PROCEEDINGS OF THE

AMERICAN MATHEMATICAL SOCIETY

Volume 138, Number 1, January 2010, Pages 263-265

S 0002-9939(09)10077-1

Article electronically published on September 4, 2009

\title{
ON THE EVANS-KRYLOV THEOREM
}

\author{
LUIS CAFFARELLI AND LUIS SILVESTRE
}

(Communicated by Matthew J. Gursky)

\begin{abstract}
We provide a short proof of the $C^{2, \alpha}$ interior estimate for convex fully nonlinear elliptic equations. This result was originally proved by L. C. Evans and N. Krylov. Our proof is based on the ideas from our work on integro-differential equations.
\end{abstract}

The Evans-Krylov theorem consists of the following a priori estimate.

Theorem 1. Smooth solutions, $u$, of a uniformly elliptic, fully nonlinear convex equation $F\left(D^{2} u\right)=0$ in the unit ball $B_{1}$, of $\mathbb{R}^{n}$ have a $C^{2, \alpha}$ interior a priori estimate

$$
\|u\|_{C^{2, \alpha}\left(B_{1 / 2}\right)} \leq C\|u\|_{C^{1,1}\left(B_{1}\right)}
$$

with the constant $C$ depending only on the ellipticity of $F$.

The importance of the Evans-Krylov theorem is that it allows us to solve the Dirichlet problem for fully nonlinear equations by the method of continuity (rendering classical solutions).

This theorem was proved independently by N. Krylov [4] and L. C. Evans [3]. In this note, motivated by our work on integral fully nonlinear equations [1], we provide a more direct presentation of their proof (although the underlying key ideas are the same).

We recall the two opposite components in Krylov-Safonov Harnack inequality, the proof of which can be found in 2] (Theorem 4.8).

a) (The weak $L^{\varepsilon}$ estimate) If $v$ is a nonnegative supersolution of

$$
a_{i j}(x) D_{i j} v \leq 0
$$

in $B_{1}$, with $\lambda I \leq a_{i j} \leq \Lambda I$, then

$$
\left|\left\{v>t \inf _{B_{1 / 2}} v\right\} \cap B_{1 / 4}\right| \leq C(\lambda, \Lambda) t^{-\varepsilon} .
$$

b) (The oscillation lemma) If $v$ is a subsolution of $a_{i j}(x) D_{i j} v \geq 0$ in $B_{1}$,

$$
0 \leq v \leq 1
$$

then

$$
\sup _{B_{1 / 2}} v \leq C(\lambda, \Lambda)\left|\{v>0\} \cap B_{3 / 4}\right| .
$$

Received by the editors May 8, 2009.

2000 Mathematics Subject Classification. Primary 35J60.

(C)2009 American Mathematical Society 
In case of harmonic functions, these are just consequences of the mean value theorem.

We also recall that convexity of $F$ as a function of $D^{2} u$, implies that any pure second derivative, $u_{\sigma \sigma}$, of $u$ and thus any linear combination

$$
\ell(x)=\sum_{j} u_{\sigma_{j} \sigma_{j}}(x)
$$

is a supersolution of the linearized operator

$$
a_{i j}(x) D_{i j} \ell(x) \leq 0
$$

$\left(a_{i j}(x)=F_{i j}\left(D^{2} u(x)\right)\right)$.

Finally, the uniform ellipticity of $F$ implies that for any two points $x_{1}, x_{2}$ in $B_{1}$,

$$
\operatorname{tr}\left[D^{2} u\left(x_{2}\right)-D^{2} u\left(x_{1}\right)\right]^{+} \approx \operatorname{tr}\left[D^{2} u\left(x_{2}\right)-D^{2} u\left(x_{1}\right)\right]^{-} .
$$

At this point, we define for any subspace $V$

$$
w(x, V)=\Delta_{V} u(x)-\Delta_{V} u(0)
$$

$\left(\Delta_{V} u(x)\right.$ is the Laplacian of $u$ at the point $x$ when restricted to the affine variety $x+V)$.

Note that for each fixed $V, w$ is an $\ell(x)$ as above and satisfies the $L^{\varepsilon}$ estimate. Also, note that the positive and negative part of the laplacian can be expressed as

$$
\begin{aligned}
& \max _{V} w(x, V)=\operatorname{tr}\left[D^{2} u(x)-D^{2} u(0)\right]^{+}, \\
& \min _{V} w(x, V)=-\operatorname{tr}\left[D^{2} u(x)-D^{2} u(0)\right]^{-} .
\end{aligned}
$$

By rescaling dyadically and iterating, it is enough to prove the following lemma.

Lemma 2. There exists a $\theta>0, \theta=\theta(\lambda, \Lambda)$, such that if for all $V$, for all $x$ in $B_{1}$

$$
w(x, V) \geq-1 .
$$

Then for all $V$, for all $x$ in $B_{1 / 2}$,

$$
w(x, V) \geq-1+\theta .
$$

Indeed, this will imply by iteration that the laplacian is Hölder continuous. Now we prove the lemma.

Proof. Assume that $w\left(x_{0}, V_{0}\right) \leq-1+\theta$ for some $V_{0}$ and $x_{0}$ in $B_{1 / 2}(\theta$, small, to be chosen). We will then find a contradiction. Since $w(\cdot, V)+1$ is a nonnegative supersolution, the $L^{\varepsilon}$ lemma applies and

$$
w(x, V)+1 \leq \theta^{1 / 2}
$$

in a set $\Omega$ that covers almost all of $B_{1 / 4}$, i.e.,

$$
\left|B_{1 / 4} \backslash \Omega\right| \leq C \theta^{\varepsilon / 2} \text {. }
$$

We notice that in $\Omega, 1-\theta^{1 / 2} \leq-w(x, V) \leq \operatorname{tr}\left[D^{2} u(x)-D^{2} u(0)\right]^{-} \leq 1$. On the other hand, we know that

$$
\begin{aligned}
w(x, V)+w\left(x, V^{\perp}\right) & =\triangle u(x)-\triangle u(0) \\
& =\operatorname{tr}\left[D^{2} u(x)-D^{2} u(0)\right]^{+}-\operatorname{tr}\left[D^{2} u(x)-D^{2} u(0)\right]^{-} .
\end{aligned}
$$


Thus, we also have $0 \leq \operatorname{tr}\left[D^{2} u(x)-D^{2} u(0)\right]^{+}-w\left(x, V^{\perp}\right) \leq \theta^{1 / 2}$ for $x \in \Omega$. Moreover, for $\theta$ small, by (11),

$$
-w(x, V) \approx \operatorname{tr}\left[D^{2} u(x)-D^{2} u(0)\right]^{-} \approx \operatorname{tr}\left[D^{2} u(x)-D^{2} u(0)\right]^{+} \approx w\left(x, V^{\perp}\right) .
$$

Thus, there is a constant $c(\lambda, \Lambda)>0$ such that $w\left(x, V^{\perp}\right) \geq c(\lambda, \Lambda)$ in $\Omega$. We now examine the function $v=\left(c(\lambda, \Lambda)-w\left(x, V^{\perp}\right)\right)^{+}$in $B_{1 / 4}$, for which the oscillation lemma applies and satisfies

a) $0 \leq v \leq 2$,

b) $v(0)=c(\lambda, \Lambda)$,

c) $v=0$ in $\Omega$.

For $\theta$ small (i.e., for $\Omega$ almost all of $B_{1 / 4}$ ) this contradicts the oscillation lemma since $c(\lambda, \Lambda)$ is a fixed positive constant for $\theta$ small. This completes the proof.

\section{ACKNOWLEDGMEnts}

Both authors were partially supported by NSF grants.

\section{REFERENCES}

[1] L. Caffarelli and L. Silvestre. The Evans-Krylov theorem for nonlocal fully nonlinear equations. Preprint.

[2] L. A. Caffarelli and X. Cabre. Fully nonlinear elliptic equations. Amer. Math. Soc. Colloq. Publ., 43, American Mathematical Society, Providence, RI, 1995. MR.1351007 (96h:35046)

[3] Lawrence C. Evans. Classical solutions of fully nonlinear, convex, second-order elliptic equations. Comm. Pure Appl. Math., 35(3):333-363, 1982. MR649348 (83g:35038)

[4] N. V. Krylov. Boundedly inhomogeneous elliptic and parabolic equations. Izv. Akad. Nauk SSSR Ser. Mat., 46(3):487-523, 670, 1982. MR661144 (84a:35091)

Department of Mathematics, University of Texas at Austin, 1 University Station C1200, Austin, Texas 78712-0257

E-mail address: caffarel@math.utexas.edu

Department of Mathematics, University of Chicago, Chicago, Illinois 60637

E-mail address: luis@math.uchicago.edu 